\title{
ARTICLE
}

\section{Overview of the PHITS code and its application to medical physics}

\author{
Tatsuhiko Sato ${ }^{a^{*}}$, Koji Niita ${ }^{\mathrm{b}}$, Norihiro Matsuda ${ }^{\mathrm{a}}$, Shintaro Hashimoto ${ }^{\mathrm{a}}$, Yosuke Iwamoto ${ }^{\mathrm{a}}$, Shusaku Noda ${ }^{\mathrm{a}}$, \\ Hiroshi Iwase ${ }^{\mathrm{c}}$, Hiroshi Nakashima ${ }^{\mathrm{a}}$, Tokio Fukahori ${ }^{\mathrm{a}}$, Satoshi Chiba ${ }^{\mathrm{a}, \mathrm{d}}$ and Lembit Sihver ${ }^{\mathrm{e}}$ \\ ${ }^{a}$ Japan Atomic Energy Agency, Shirakata-Shirane 2-4, Tokai, Ibaraki, 319-1195, Japan; ${ }^{b}$ Research Organization for Information \\ Science and Technology, Shirakata-Shirane 2-4, Tokai, Ibaraki, 319-1106, Japan; ' $H i g h$ Energy Accelerator Research \\ Organization, Oho 1-1, Tsukuba, Ibaraki, 305-0801, Japan; ${ }^{d}$ Tokyo Institute of Technology, Ookayama 2-12-1, Meguro, Tokyo, \\ 152-8550, Japan; ${ }^{e}$ Chalmers University of Technology, Göteborg SE-412 96, Sweden
}

\begin{abstract}
Particle and Heavy Ion Transport code System, PHITS, is a general purpose three-dimensional Monte Carlo particle transport simulation code developed through collaboration with several institutes in Japan and Europe. To apply PHITS to medical physics, two special functions were implemented in the code: an event generator mode and a microdosimetric tally function. Utilizing these functions, a new relative-biological-effectiveness -weighted dose estimation method for charged-particle therapy was established on the basis of the double-stochastic microdosimetric kinetic model. Owing to these features, PHITS has been used for various medical applications, such as patient dose estimation for radiotherapy and computed tomography examination.
\end{abstract}

Keywords: PHITS; Monte Carlo; event generator; medical physics; RBE-weighted dose; microdosimetry

\section{Introduction}

Currently, Monte Carlo codes for particle transport simulation in three-dimensional matters are indispensable for various applications, such as radiation shielding design and medical physics. Therefore, we are developing a general purpose Monte Carlo Particle and Heavy-Ion Transport code System, PHITS [1], through collaboration with several institutes in Japan and Europe. Figure 1 shows the institutes that are currently contributing to the development of PHITS. The Japan Atomic Energy Agency (JAEA) is responsible for managing the entire project. The code has been distributed to many countries via the Research organization for Information Science and Technology, the Data Bank of the Organization for Economic Co-operation and Development's Nuclear Energy Agency, and the Radiation Safety Information Computational Center.

PHITS can deal with the transport of nearly all particles, including neutrons, protons, heavy ions, photons, and electrons, over wide energy ranges using several nuclear reaction models and nuclear data libraries. The geometrical configuration of the simulation can be set with general geometry (GG) or combinatorial geometry (CG). Various quantities, such as heat deposition, track length, and production yields, can be deduced from the PHITS simulation using implemented "tally" estimator functions.

To apply PHITS to medical physics, two special

*Corresponding author. Email: sato.tatsuhiko@jaea.go.jp functions were implemented in the code: an event generator mode [2,3] and a microdosimetric tally function [4,5]. Utilizing these functions, we established a computational model for calculating the relative biological effectiveness (RBE)-weighted dose for charged particle therapy [6]. The features of these functions and the model are described in this paper. Other examples of the application of PHITS to medical physics are also introduced herein.

\section{Special features}

\subsection{Event generator mode}

There are two types of Monte Carlo methods for simulating nuclear reactions: "event generators" and "non-event generators." The former conserves the energy and momentum before and after a reaction called the "event," whereas the latter does not. Most nuclear

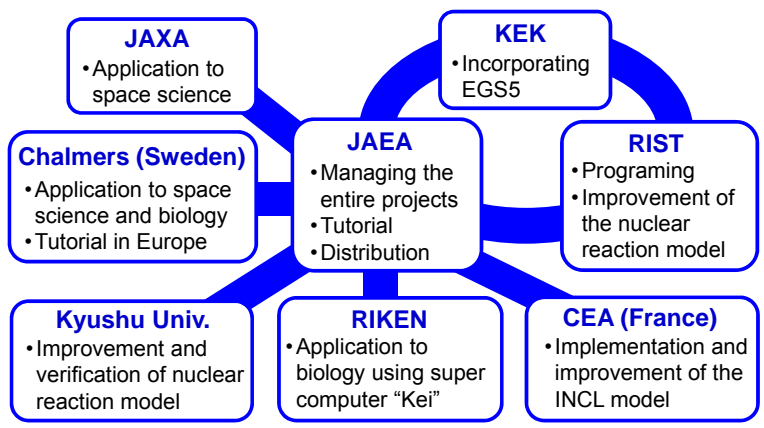

Figure 1. Institutes currently contributing to the development of PHITS. Blue lines indicate the contracts between each institute. 


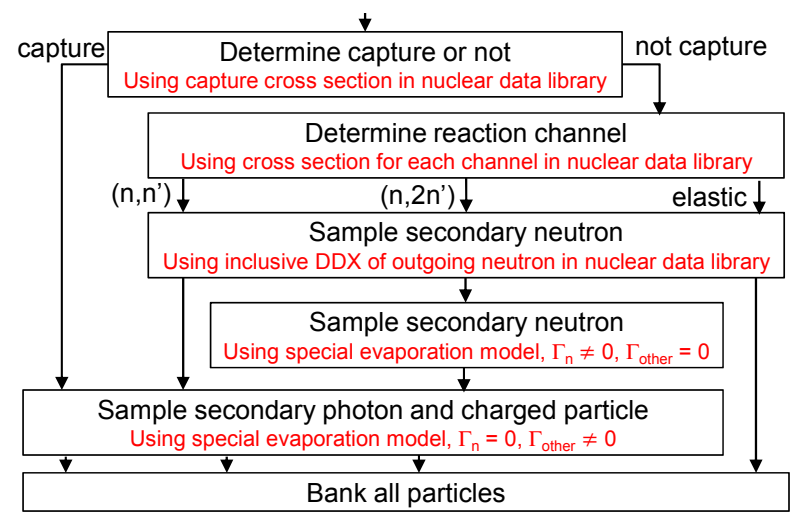

Figure 2. Basic flowchart of the event generator mode in PHITS for simulating low-energy neutron-induced reaction.

reaction models, such as the intra-nuclear cascade model, are "event generators", but Monte Carlo simulations using nuclear data libraries are generally "non-event generators," because only inclusive cross-section data are contained in the libraries. For example, the sum of the energies of two neutrons emitted from an $(n, 2 n$ ') reaction is occasionally greater than the incident energy, because the energies of outgoing particles are independently sampled using the inclusive cross section. Thus, only the mean values can be deduced from "non-event generator" simulations. However, it is occasionally necessary to estimate the distribution around the mean value, such as for the response function of detectors and the single-event upset probability of semiconductor devices.

In addition, the energy and momentum of residual nuclides cannot be estimated from "non-event generator" simulations, because they are generally determined by calculating the difference between the initial and final states; that means, conservation of energy and momentum must be established in an event. This information is indispensable for estimating the radiation risk of neutron exposure in medical physics and space dosimetry, because the radiation quality is generally expressed by the profiles of not neutrons but secondary charged particles emitted from neutron-induced reactions.

Based on these considerations, we implemented a unique "event generator mode" in PHITS for simulating low-energy neutron-induced reactions using nuclear data libraries. A special evaporation model was developed for this purpose to maintain conservation of energy and momentum in an event. The basic flowchart is shown in Figure 2. Any observables, such as the energy and momentum of residual nuclides, can be deduced using this mode. Thus, PHITS can directly calculate the dose equivalent based on the $Q(L)$ relationship, even for neutron irradiation. This feature is very important for applying the code to the risk estimation for astronauts, because the dose equivalent is frequently used as a surrogate for the equivalent dose in space dosimetry.

\subsection{Microdosimetric Tally Function}

Linear energy transfer (LET) is a widely used index for expressing the radiation quality of charged particles. However, the radiation quality of high-energy and -charge (HZE) particles cannot be uniquely determined from their LET because of the differences in the ionizing densities around their trajectories, resulting from the large cross sections of high-energy $\delta$-ray production.

Microdosimetric quantities, such as the lineal energy, $y$, and the specific energy, $z$, as defined in ICRU Report 36 [7], are considered to be better indexes for expressing the radiation quality of HZE particles because they are directly related to the ionizing densities in microscopic sites. Their probability density (PD) around the trajectory of a given mono-energetic particle can be calculated using track-structure simulation codes, which explicitly handle ionization and excitation event-by-event. However, it used be very difficult to evaluate the microdosimetric PD for macroscopic matter, because event-by-event analysis of ionization and excitation is extremely time-consuming in comparison to condensed history simulation, which is generally employed in macroscopic particle transport simulation codes such as PHITS.

Therefore, we established a new tally function in PHITS for estimating microdosimetric PDs for macroscopic matter within a reasonable computational time [5]. In this tally, the deposition energies in macroscopic matter are scored as a function of the charge and energy of the particles and then are converted into microdosimetric PDs using a mathematical function that can instantaneously calculate the PDs around the trajectory of any type of charged particle [4]. This mathematical function was developed on the basis of the least-squares fitting of the PDs, calculated using a track-structure simulation code for several mono-energetic charged particles. The diameter of the microdosimetric site can be arbitrarily specified from $1 \mathrm{~nm}$ to $2 \mu \mathrm{m}$.

The accuracy of this calculation method was well verified by comparing the results with corresponding data measured using a wall-less tissue-equivalent proportional counter [8]. This tally function was used for calculating the organ dose equivalents based on the $Q(y)$ relationship $[9,10]$ and the RBE-weighted dose estimation, as described in the next section.

\section{Applications of PHITS to medical physics}

\subsection{RBE-weighted dose estimation}

The RBE-weighted dose was introduced for estimating the relative therapeutic effect of charged-particle therapy. It is defined as the product of the absorbed dose and the relative biological effectiveness. Most clinical centers where proton therapy is administered adopt a constant RBE of 1.1 for estimating the RBE-weighted dose. On the other hand, RBEs for heavy-ion therapy vary more dramatically with the irradiation conditions, and their numerical 


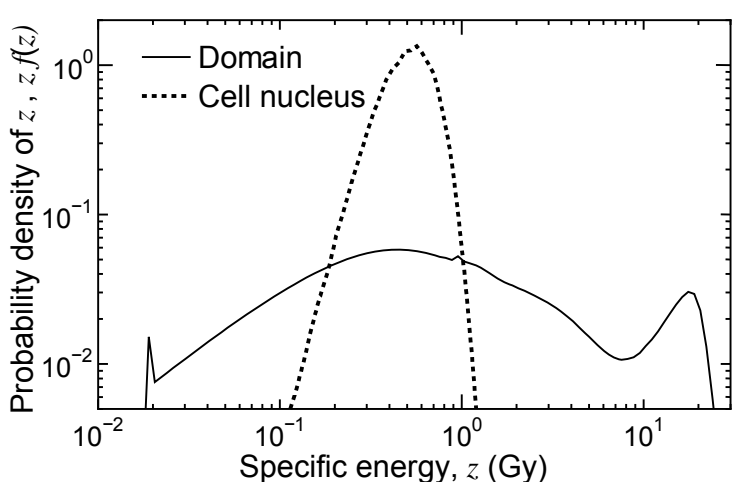

Figure 3. PDs of the specific energies in domains and cell nuclei irradiated with $135 \mathrm{MeV} / \mathrm{u}$ carbon ions at $0.5 \mathrm{~Gy}$, calculated using PHITS in combination with the convolution method.

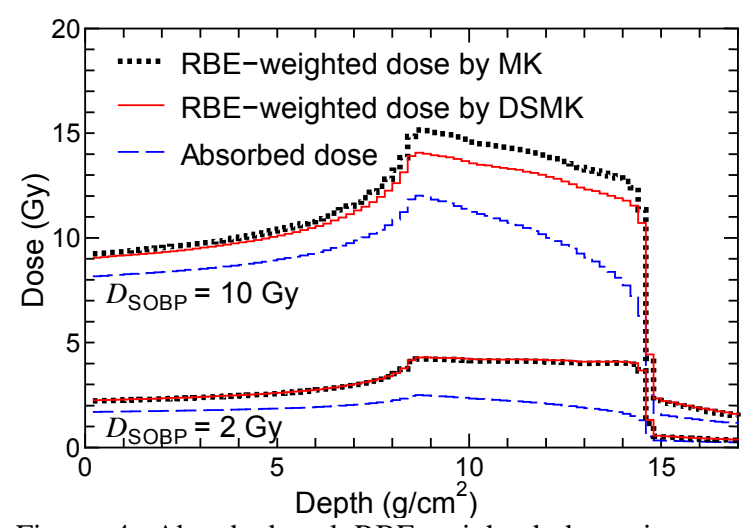

Figure 4. Absorbed and RBE-weighted doses in a water phantom irradiated with a carbon $290 \mathrm{MeV} / \mathrm{u}$ spread-out Bragg peak (SOBP) beam calculated using PHITS coupled with the MK and DSMK models. The absorbed doses at the center of the SOBP, $D_{\text {SoBp}}$, were adjusted to 2 and 10 Gy for lower and higher dose irradiations, respectively.

values are estimated using models developed on the basis of the results of in vitro experiments on cell-survival fractions.

Therefore, we established an original RBE-weighted dose estimation method using PHITS coupled with the double stochastic microdosimetric kinetic (DSMK) model [6], which enables the calculation of the cell-survival fractions for any irradiation condition based on the PD of the specific energy in the cell nucleus as well as its substructure called “domain.” The DSMK model is an improved version of the microdosimetric kinetic (MK) model [11], which simply represents the PD of the cell-nucleus specific energy with its mean value; i.e. the inhomogeneity of the doses in each cell nucleus is not considered in the original MK model. This ignorance leads to inaccurate estimation of cell-survival fractions for high-LET and high-dose irradiations.

In the RBE-weighted dose estimation method based on the DSMK model, the PDs of the domain and cell-nucleus specific energies are calculated using the microdosimetric and LET-estimator tally functions implemented in PHITS in combination with the convolution or database method. Figure 3 shows examples of calculated PDs for specific energies in domains and cell-nuclei irradiated using $135 \mathrm{MeV} / \mathrm{u}$ carbon ions at $0.5 \mathrm{~Gy}$. The domains and cell nuclei are assumed to be spherical water with radii of 0.27 and 6.2 $\mu \mathrm{m}$, respectively. It can be clearly seen that the PDs are broadened from their average values, particularly for the domain specific energy. This broadening occurs because domains are much smaller than cell nuclei, and only a few percent of them are directly irradiated by the primary ions, whose contribution is observed in the peak near 20 Gy. In the DSMK model, the cell-survival fractions can be determined from this inhomogeneity of doses by solving mathematical equations derived from the theory of dual radiation action [12].

Figure 4 shows the absorbed and RBE-weighted doses in a water phantom irradiated with a carbon 290 $\mathrm{MeV} / \mathrm{u}$ spread-out Bragg peak (SOBP) beam calculated using PHITS coupled with the MK and DSMK models. The RBE-weighted doses obtained from the two models are nearly identical for lower-dose irradiation, while the DSMK model gives smaller values for the higher-dose irradiation. This tendency indicates that the consideration of the stochastic nature of the cell-nucleus specific energy, which is ignored in the original MK model, is very important in the treatment planning of hypo-fractionated charged-particle therapy.

\subsection{Other applications}

Several other applications of PHITS to medical physics are introduced in this section. Nose et al. succeeded in reproducing the depth- and lateral-dose distributions of SOBP carbon-ion beams by implementing two functions into PHITS, including one for energy dispersion calculations and another for transport in an AC magnetic field [13]. The consideration of the angular straggling of charged particles is also indispensable for this calculation. Using these functions, Nose et al. analyzed the field-size effect of radiation quality in carbon-ion therapy [14] and found that the dose and radiation quality at the center of the broad beam are influenced by particles from the off-center region via large-angle scattering. Yonai et al. calculated the absorbed-dose and dose-equivalent distributions far from the target organs for estimating the secondary cancer risk of carbon-ion therapy, using the event generator model [15].

Fujibuchi et al. employed PHITS for analyzing the neutron fields in 10 and 15 MV electron linac facilities used for X-ray radiotherapy [16]. This study was motivated by recent concerns regarding radiological protection against neutrons generated by photo-nuclear reactions in those facilities. They found that the dose contribution from neutrons is negligibly small in comparison to that from photons in a $10 \mathrm{MV}$ linac facility, but dramatically increases with the accelerated electron energy. It should be mentioned that the current version of PHITS can deal with only giant resonances in photo-nuclear reaction mechanisms, which have the largest cross section for photons less than $20 \mathrm{MeV}$. The implementation of higher-energy photo-nuclear reaction 
mechanisms, such as quasi-deuteron disintegration, is currently in progress.

Takahashi et al. developed a database of organ doses from computed tomography (CT) examinations using PHITS coupled with a Japanese male voxel phantom [17]. The results were compared with corresponding data obtained using a stylized phantom. It was found that the doses in some organs calculated using the voxel and stylized phantoms were different because of the differences in their anatomies. Their database is implemented in WAZA-ARI, which is a web system developed for assessing the organ doses for the CT examination of individual patients.

\section{Conclusion}

PHITS has been used in various medical applications owing to its special functions, such as the event generator mode and the microdosimetric tally function. Several projects that apply PHITS for practical medical purposes are currently in progress, such as the European Union FP7 project ALLEGRO [18]. Development of a PHITS-based treatment planning system has also been initiated on the basis of our established RBE-weighted dose estimation method.

\section{Acknowledgements}

The authors wish to thank Dr. H. Nose of IHI Corporation, Dr. T. Fujibuchi of Ibaraki Prefectural University of Health Sciences, and Dr. F. Takahashi of JAEA for their help in preparing this manuscript.

\section{References}

[1] K. Niita, N. Matsuda, Y. Iwamoto, H. Iwase, T. Sato, H. Nakashima, Y. Sakamoto and L. Sihver, PHITS: Particle and Heavy Ion Transport Code System, Version 2.23, JAEA-Data/Code, Japan Atomic Energy Agency, 2010-022 (2010).

[2] Y. Iwamoto, K. Niita, Y. Sakamoto, T. Sato and N. Matsuda, Validation of the event generator mode in the PHITS code and its application, Int. Conf. on Nuclear Data for Science and Technology 2007, (2007) DOI: 10.1051/ndata:07417.

[3] K. Niita, Y. Iwamoto, T. Sato, H. Iwase, N. Matsuda, Y. Sakamoto and H. Nakashima, A new treatment of radiation behavior beyond one-body observables, Int. Conf. on Nuclear Data for Science and Technology 2007, (2007) DOI: 10.1051/ndata:07398.

[4] T. Sato, R. Watanabe and K. Niita, Development of a calculation method for estimating the specific energy distribution in complex radiation fields, Radiat. Prot. Dosim. 122 (2006), pp. 41-45.

[5] T. Sato, Y. Kase, R. Watanabe, K. Niita and L. Sihver, Biological dose estimation for charged-particle therapy using an improved PHITS code coupled with a microdosimetric kinetic model, Radiat. Res. 171 (2009) , pp. 107-117.

[6] T. Sato and Y. Furusawa, Cell-survival fraction estimation based on the probability densities of domain and cell-nucleus specific energies using improved microdosimetric kinetic models, Radiat. Res. 178 (2012), pp 341-356.

[7] International Commission on Radiation Units and Measurements, Microdosimetry, ICRU Report 36, Bethesda, Maryland, USA (1983).

[8] S. Tsuda, T. Sato, F. Takahashi, D. Satoh, A. Endo, S. Sasaki, Y. Namito, H. Iwase, S. Ban and M. Takada, Measurement of microdosimetric spectra with a wall-less tissue-equivalent proportional counter for a $290 \mathrm{MeV} / \mathrm{u}{ }^{12} \mathrm{C}$ beam, Phys. Med. Biol. 55 (2010), pp. 5089-5101.

[9] T. Sato, A. Endo, M. Zankl, N. Petoussi-Henss and K. Niita, Fluence-to-dose conversion coefficients for neutrons and protons calculated using the PHITS code and ICRP/ICRU adult reference computational phantoms, Phys. Med. Biol. 54 (2009), pp. 1997-2014.

[10] T. Sato, A. Endo and K. Niita, Fluence-to-dose conversion coefficients for heavy ions calculated using the PHITS code and ICRP/ICRU adult reference computational phantoms, Phys. Med. Biol. 55 (2010), pp. 2235-2246.

[11] R.B. Hawkins, A statistical theory of cell killing by radiation of varying linear energy transfer, Radiat. Res. 140 (1994), pp. 366-374.

[12] A.M. Kellerer and H.H. Rossi, A generalized formation of dual radiation action, Radiat. Res. 75 (1978), pp. 471-488.

[13] H. Nose, K. Niita, M. Hara, K. Uematsu, O. Azuma, Y. Miyauchi, M. Komori and T. Kanai, Improvement of three-dimensional Monte Carlo code PHITS for heavy ion therapy, J. Nucl. Sci. Technol. 42 (2005), pp. 250-255.

[14] H. Nose, Y. Kase, N. Matsufuji and T. Kanai, Field size effect of radiation quality in carbon therapy using passive method, Med. Phys. 36 (2009), pp. 870-875.

[15] S. Yonai, N. Matsufuji and M. Namba, Calculation of out-of-field dose distribution in carbon-ion radiotherapy by Monte Carlo simulation, Med. Phys. 39 (2012), pp. 5028-5039.

[16] T. Fujibuchi, S. Obara, H. Sato, M. Nakajima, N. Kitamura, T. Sato, H. Kumada, T. Sakae and T. Fujisaki, Estimate of photonuclear reaction in a medical linear accelerator using a water-equivalent phantom. Prog. Nucl. Sci. Technol. 2 (2011), pp. 803-807.

[17] F. Takahashi, K. Sato, A. Endo, K. Ono, T. Yoshitake, T. Hasegawa, Y. Katsunuma, N. Ban and M. Kai, WAZA-ARI: computational dosimetry system for X-ray CT examinations. I. Radiation transport calculation for organ and tissue doses evaluation using JM phantom, Radiat. Prot. Dosim. 146 (2011), pp. 241-243.

[18] The ALLEGRO project, Early and late health risk to normal/healthy tissues from the use of existing and emerging techniques for radiation therapy, http://allegroproject.org/ (accessed on Dec. 1, 2012). 